\title{
Effect of saturated/unsaturated phosphatidylcholine ratio on the stability of liposome-encapsulated hemoglobin
}

\author{
Yi-You Huang a,*, Tze-Wen Chung ${ }^{\mathrm{b}}$, Cheng-I Wu \\ ${ }^{a}$ Institute of Biomedical Engineering, College of Medicine, National Taiwan University, Taipei, Taiwan \\ ${ }^{\mathrm{b}}$ Department of Biomedical Engineering, Chung-Yuan University, Chung-Li, Taiwan
}

Received 24 February 1998; received in revised form 27 May 1998; accepted 9 June 1998

\begin{abstract}
The influence of different combinations of saturated and unsaturated phosphatidylcholines (PCs) as well as addition of $\alpha$-tocopherol on lipid peroxidation and particle size change was analyzed. Because unsaturated egg PC was susceptible to peroxidation, partially hydrogenated egg PC or hydrogenated egg PC (H-PC) was frequently used instead of egg PC. The efficacy of another alternative with combinations of saturated and unsaturated PCs was investigated. Oxidation can be inhibited by adding $\alpha$-tocopherol in the lipid formulation or by using different combinations of H-PC and PC. Adding $\alpha$-tocopherol was shown to cause physical instability of liposomes, and increase the possibility of aggregation. Increasing the H-PC content in the liposome formulation will effectively reduce the peroxidation. The stability of liposome was dependent on both $\alpha$-tocopherol and H-PC to PC ratio. Encapsulating hemoglobin in the liposome would promote the lipid peroxidation. (C) 1998 Elsevier Science B.V. All rights reserved.
\end{abstract}

Keywords: LEH; Liposome; Peroxidation

\section{Introduction}

Research into artificial oxygen-carrying blood substitutes has been ongoing for many years (Chang, 1993; Brandl and Gregoriadis, 1994). Liposome-encapsulated hemoglobin (LEH) has

\footnotetext{
* Corresponding author. Fax: +886223940049.
}

been shown to be a promising approach as an oxygen-carrying red cell substitute, and also has been shown to be beneficial in the treatment of hemorrhagic shock (Hunt et al., 1985; Rollwagen et al., 1996). The success of the use of liposomes as drug carriers or oxygen-carrying blood substitutes depends on their stability in blood stream following intravenous infusion. In our earlier 
study, $10-20 \%$ of encapsulated hemoglobin in LEH would be released when it was subjected into a defined flow field (Chung et al., 1997). It suggested that hemoglobin may be released to blood stream and possibly promote free radical reaction through Fenton or other reactions (John, 1986; Winterbourn, 1995). The attack of free radical from blood stream or suspension media, in this study, to the membrane of liposomes may possibly reduce the circulating time of liposome or LEH in the circulation system. In the other hand, physical and/or chemical instability of certain aqueous liposome dispersions during storage causes difficulties in their formulation as pharmaceutically acceptable products. Therefore, as a part of pharmaceutical formulation studies, assessment and manipulation of chemical and physical stability of liposome are important issues.

Liposomes can be formulated by many alternative preparation techniques and using different formulations. Conventional liposomes made from natural lecithin are prone to physical and chemical deterioration, such as aggregation and peroxidation. To reduce the oxidative interactions between hemoglobin and membrane lipid of LEH, SOD/catalase, cholesterol and/or antioxidants, were usually added to the hemoglobin suspensions (Zheng et al., 1994). The antioxidant, $\alpha$-tocopherol (vitamin E), was frequently incorporated into the liposome to inhibit the oxidation of lipid (Hunt and Tsang, 1981; Konings, 1984). Hydrogenated PC or partially hydrogenated PC instead of unsaturated egg PC in liposome preparation has been widely used to reduce lipid peroxidation (Farmer and Gaber, 1987; New, 1990).

Since the cost of hydrogenated PC is much higher than that of egg PC, it may be valuable to reduce the cost in producing LEH by reducing the quantities of hydrogenated PC in forming LEH with same oxygen carrying capacity. To evaluate this issue, in this study, we investigated the effect of the unsaturated/saturated PC ratio on the stability of liposomes or LEH and the influence of adding $\alpha$-tocopherol on the size change of liposomes.

\section{Materials and methods}

\subsection{Materials}

Egg yolk PC (Type X-E, 60\% phosphatidylcholine), hydrogenated egg yolk PC (Type I-EH, highly purified egg lecithin hydrogenated in the presence of a platinum catalyst, 99\% phosphatidylcholine), cholesterol, $\alpha$-tocopherol, protamine and phosphate buffer saline were purchased from Sigma (St. Louis, MO). Chloroform, methanol, $n$-butanol and other chemicals used in the experiments and analysis were reagent grade (Wako, Japan).

Stroma free haemoglobin (SFHb) was extracted from human packed erythrocytes by the two-stage aqueous two-phase system and subsequently was desalted and dialyzed by ultrafiltration (Kan and Lee, 1994; Lee and Kan, 1995). The high purity of $\mathrm{SFHb}$ was obtained. Oxidation of $\mathrm{Hb}$ during storage at $4^{\circ} \mathrm{C}$ was suppressed.

\subsection{Preparation of blank liposomes and LEH}

Different liposome compositions were evaluated according their physical and chemical stability. The compositions used were (in mole ratio): total phospholipid:cholesterol (CHOL) (1:1), where the phospholipids were the combinations of different ratios of egg phosphatidylcholine (PC) and hydrogenated egg phosphatidylcholine $(\mathrm{H}-$ PC), e.g. $\mathrm{PC}: \mathrm{H}-\mathrm{PC}=10: 0, \quad 9: 1, \quad 8: 2, \quad 7: 3, \quad 5: 5$, $1: 1.25$. In some cases, $\alpha$-tocopherol was added at the mole ratio of total phospholipid:CHOL: $\alpha$-tocopherol $=1: 1: 0.1$. The liposomes were obtained by mechanical disruption of lamellar phases by sonication, followed by extrusion through polycarbonate membranes of $0.2 \mu \mathrm{m}$ pore size. Phospholipid, CHOL and/or $\alpha$-tocopherol were dissolved in chloroform/ethanol $(1 \% \mathrm{v} / \mathrm{v})$. The organic solvent of the lipid solution was removed with a rotary vacuum evaporator (EYELA N1NW, Tokyo, Japan), then further purged with nitrogen. The lipids were dispersed in PBS by sonication or by vortexing, and multilamellar liposomes (MLV) was formed. To encapsulate $\mathrm{Hb}$ in phospholipid, $\mathrm{Hb}$ solution $(10 \mathrm{~g} / \mathrm{dl})$ of was used instead of PBS. The MLV was further sonicated 
(150 W, Probe Ultrasonic Processor GE50T, Cole-Parmer, IL) at $4^{\circ} \mathrm{C}$ for 30 min or extruded through polycarbonate membranes (Nucleopore, CA) of $0.2 \mu \mathrm{m}$ pore size to reduce the vesicle size. The mixture was then centrifuged $(20000 \times g$ at $4^{\circ} \mathrm{C}$ ) for $15 \mathrm{~min}$ to sediment undispersed lipid and MLVs. Untrapped hemoglobin (supernatant) was discarded after protamine was added to the solution followed by ultra-centrifugation $(20000 \times g$ at $4^{\circ} \mathrm{C}$ for $30 \mathrm{~min}$ ). The $\mathrm{LEH}$ or liposome sediment was re-suspended by adding heparin solution to the desired concentration. Hematocrit of LEH was approximate to $40 \%$. In all experiments in this study, except specially mentioned, liposomes were formulated with egg PC and H-PC only, without adding $\alpha$-tocopherol or encapsulating any biologically active materials. LEH meant liposome-encapsulated hemoglobin without adding antioxidants.

The physical stability was checked at $4^{\circ} \mathrm{C}$, by determining the changes in vesicle size over a maximum of 21 days.

\subsection{Measurement of lipid peroxidation}

The degree of lipid peroxidation in liposomes was measured by the reaction between the accumulation of secondary lipid peroxidation products with 2-thiobarbituric acid (TBARS). $3 \mathrm{ml}$ of $1 \%$ phosphoric acid containing $5 \times 10^{-4} \mathrm{M}$ ethylenediaminetetraacetic acid (EDTA) and $1 \mathrm{ml}$ of a $0.5 \%$ TBA solution were added to $1 \mathrm{ml}$ of the suspension of liposomes, and the mixture then was incubated at $100^{\circ} \mathrm{C}$ for $45 \mathrm{~min}$. The samples were then cooled with subsequent addition of $4 \mathrm{ml}$ of $n$-butanol, vigorously stirred, and centrifuged at $1500 \times g$ for $15 \mathrm{~min}$. The absorption spectrum in the region from 515 to $550 \mathrm{~nm}$ was recorded in the upper butanol phase. Optical density was determined at $532 \mathrm{~nm}$, with the absorbance at 515 and $550 \mathrm{~nm}$ used as the background value. Concentrations of TBARS were presented as absorbance or as malonyldialdehyde (MDA) molar concentration, assuming the molar complex extinction coefficient of the MDA-TBA complex to be equal $1.56 \times 10^{5} / \mathrm{M}$ per $\mathrm{cm}$ (Panasenko et al., 1995).

\subsection{Incubation of liposomes with hypochlorite}

The process of peroxidation were accelerated by adding $1 \mu \mathrm{M} \mathrm{NaClO}$, with $1.5 \mathrm{mM} \mathrm{Fe}^{2+}$ in the phosphate buffer, pH 7.4 (PBS) (Panasenko et al., 1995). The intermediate product of peroxidation, MDA (malonyldialdehyde), was measure by spectrophotometer at wave length $512 \mathrm{~nm}$ (Uchiyama and Michara, 1978).

Physical stability of liposomes was monitored by measuring the size change. The process of aggregation and/or fusion of liposomes was analyzed by photon correlation spectroscopy using a Coulter Model N4 Plus sub micro particle analyzer (Hialeah, FL).

\section{Results and discussion}

Egg PC is a mixture of molecular species of PC differing in fatty acyl chains, and it includes a considerable amount of unsaturated fatty acids, such as arachidonic (C20:4) and decasohexanoic (C22:6) acids (Grit et al., 1993). These phopholipids with unsaturated acyl chains are subject to oxidation, which may affect the permeability of the bilayers and the in vivo performance of the liposomes (Smolen and Shohet, 1974; Konings, 1984). These acids are more sensitive to oxidative degradation than more saturated forms. Partially hydrogenated egg PC decreases sensitivity to oxidation (Lang et al., 1990). Many investigators used saturated (hydrogenated) PC as the major components of liposomes to reduce the possibility of peroxidation. Here, we show the effect of different combinations of saturated and unsaturated PC in the liposome formulation on particle size change and the inhibition capability during the process of peroxidation.

In the first series of experiments, the accumulation of lipid peroxidation products, reacting with 2-thiobarbituric acid (TBARS), was measured in liposomes prepared from different combinations with of PC, H-PC, and/or $\alpha$-tocopherol. It can be seen in Fig. 1 that TBARS accumulated in the course of storage at $4^{\circ} \mathrm{C}$, whereas in presence of $\alpha$-tocopherol lipid peroxidation products were virtually non-significant. This indicates that peroxi- 
dation of liposome was effectively inhibited by the addition of $\alpha$-tocopherol into the formulation. As shown in Fig. 1, without $\alpha$-tocopherol, the degree of peroxidation quickly increases during the shelf life, even in a short shelf time, as measured by the TBA method. When $\alpha$-tocopherol is added, no significant peroxidation occurs within the first two weeks, regardless of the ratio of H-PC was added. The different lipid compositions do not cause significant variation in the inhibition capability of lipid oxidation for $\alpha$-tocopherol, probably because $\alpha$-tocopherol could scavenge free radicals created between the liposomes, thus prohibiting further peroxidation of lipids.

In addition $\alpha$-tocopherol to the liposome formulation will increase the aggregation of liposomes significantly, as shown in Fig. 2. Due to the viscous properties of $\alpha$-tocopherol, liposomes incorporated with it are easily coagulated, with even a small amount of added $\alpha$-tocopherol. In addition to chemical composition and surface properties, the physical stability of liposome-based formulations, measured as changes in the particle size and distribution, as well as retention of the encapsulated biological active materials, might also be affected by the addition of $\alpha$-tocopherol.

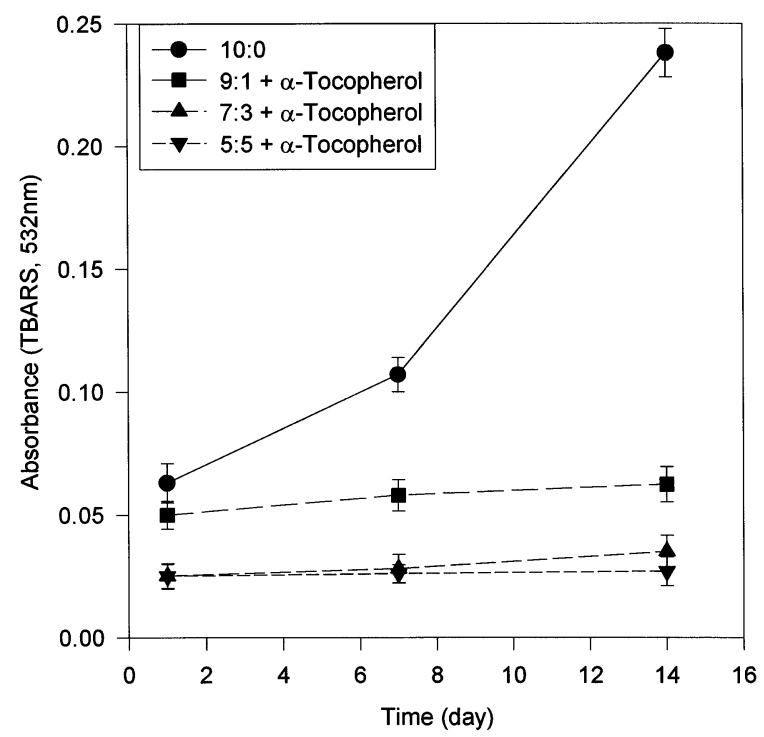

Fig. 1. Peroxidation of liposomes during shelf-life. Total lipid: $8 \mathrm{mg} / \mathrm{ml}$. 9:1 indicates ratio of egg PC:hydrogenated egg PC. (Data are the average of duplicate experiments, mean \pm S.D.)

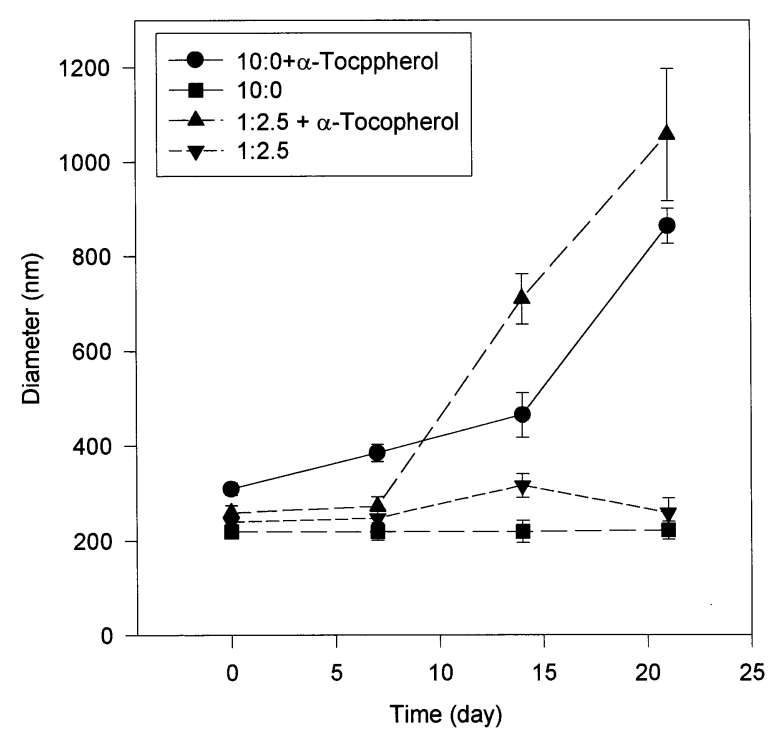

Fig. 2. Mean particle size change during shelf-life. 1:2.5 indicates ratio of egg PC:hydrogenated egg PC. (Data are the average of duplicate experiments, mean \pm S.D.)

Particle size is the most important characteristic of liposomes, affecting the drug delivery efficiency and the destiny of liposomes. Therefore, in some cases, $\alpha$-tocopherol was not used to inhibit the peroxidation in order to avoid liposome aggregation.

Fig. 2 also shows that the particle sizes of liposome made from PC or PC/H-PC 1:2.5 are almost the same. Hydrogenated PC causes little change in the liposome size compared to the changes made by conventional egg lecithin. However, they show different abilities in inhibiting lipid peroxidation. To test the inhibition ability of different formulations, $1 \mu \mathrm{M} \mathrm{NaClO}$ with 1.5 $\mathrm{mM} \mathrm{Fe}^{2+}$ was added to the PBS to accelerate the peroxidation of liposomes. The accumulation of lipid peroxidation products reacting with 2-thiobarbituric acid was measured in suspensions of liposomes prepared from different ratios of H-PC incubated with hypochlorite. The degree of peroxidation was corresponding to the MDA concentration measured. Oxidative decomposition of the lipids can be retarded by the addition of H-PC. Increasing the H-PC content in the liposome formulation will reduce the possibility of peroxidation of the liposomes (Fig. 3). Fig. 3 also shows 
the kinetics of MDA accumulation in the suspension of liposomes after addition of $1 \mu \mathrm{M}$ $\mathrm{NaClO}_{4}$ with $1.5 \mathrm{mM} \mathrm{Fe}{ }^{2+}$. When $\alpha$-tocopherol (total lipid (PC:H-PC $=10: 0$ ): CHOL: $\alpha$-tocopherol $=1: 1: 0.1)$ was added to the formulation of liposome, no significant change in the variation of MDA concentration was found even when accelerated oxidation with $1 \mu \mathrm{M} \mathrm{NaClO}{ }_{4}$ with $1.5 \mathrm{mM} \mathrm{Fe}^{2+}$ in the PBS (data not shown). Since hypochlorite induces chain reactions of lipid peroxidation, the process can be inhibited by free radical scavengers or may be retarded by the hydrogenated phosphatidylcholine. If we use the parameter of unsaturated PC to the total lipids ratio vs the degree of peroxidation, sigmoid behavior can be observed, as shown in Fig. 4. This indicates that there is a threshold of H-PC content to provide the capability to inhibit the lipid peroxidation. Only when the ratio of unsaturated PC to the total lipids is less than 0.8 , can it significantly reduce the degree of peroxidation.

Fig. 5 shows the difference of lipid peroxidation between LEH and blank liposome under the accelerated by $1 \mu \mathrm{M} \mathrm{NaClO}$, with $1.5 \mathrm{mM}$

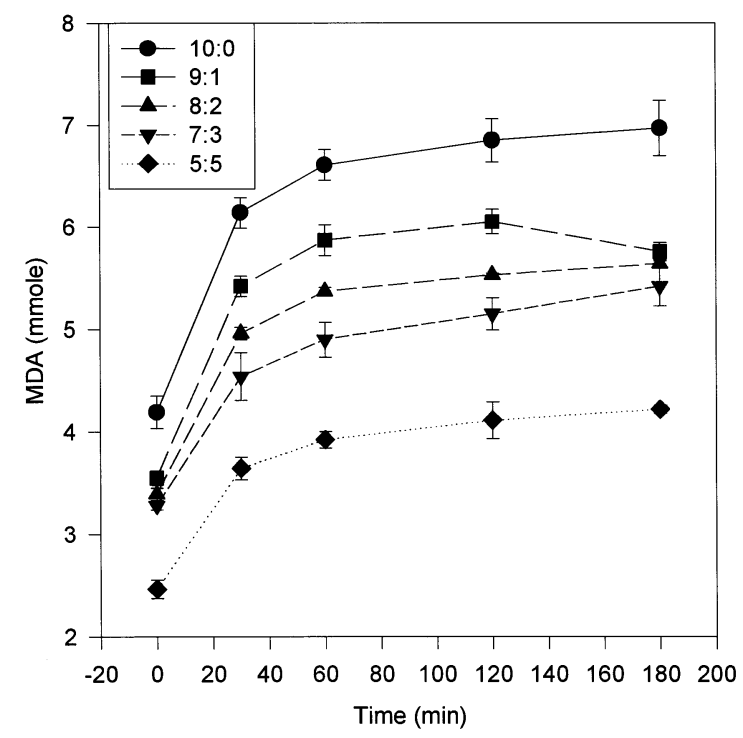

Fig. 3. Peroxidation of liposomes under the $1 \mu \mathrm{M} \mathrm{NaClO}$ with $1.5 \mathrm{mM} \mathrm{Fe}^{2+}$ in the PBS. 7:3 indicates ratio of egg PC:hydrogenated egg PC. Total lipid: $8 \mathrm{mg} / \mathrm{ml}$. (Data are the average of three consecutive experiments, mean \pm S.D.)

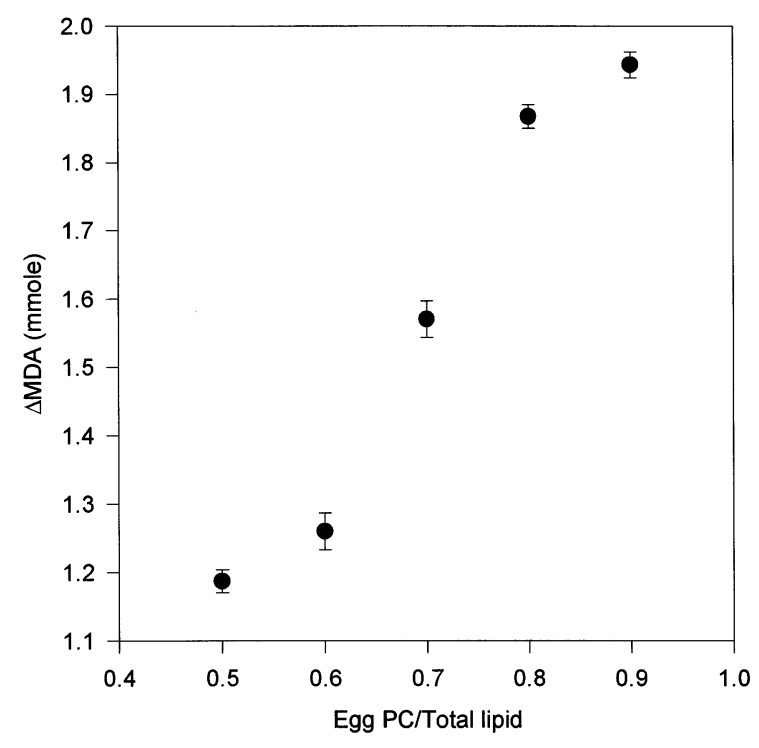

Fig. 4. Relationship of unsaturated PC/total lipid with the degree of peroxidation between the first $30 \mathrm{~min}$ in the $1 \mu \mathrm{M}$ $\mathrm{NaClO}_{4}$ with $1.5 \mathrm{mM} \mathrm{Fe}^{2+}$ in the PBS. (Data are the average of three consecutive experiments, mean \pm S.D.)

$\mathrm{Fe}^{2+}$ in the PBS. Oxidation of LEH was higher than that of blank liposomes. There are several possible reasons for the higher oxidation of $\mathrm{LEH}$, including the autoxidation of $\mathrm{Hb}$ and the complex lipid-heme interaction. Or hemoglobin in LEH might be released through the lipid bilayer to the outer suspension media and possibly promoted free radical reaction through Fenton or other reactions (John, 1986; Winterbourn, 1995), especially when it was subjected into a defined flow field (Chung et al., 1997). The resulting ferrous ions might also have the possibility to penetrate into the liposome and increase the lipid peroxidation. Furthermore, it often resulted in the oxidation of functional $\mathrm{Hb}$ to nonfunctional met-Hb. Oxidation of $\mathrm{Hb}$ to met- $\mathrm{Hb}$ may have been inhibited by using partially hydrogenated PC instead of natural unsaturated egg PC. For LEH to have a long shelf life, it is important to maintain low levels of met- $\mathrm{Hb}$ in the formulation (Deshpande and Beissinger, 1993). The mechanism of $\mathrm{Hb}$ or met-Hb on the lipid peroxidation or the effect of antioxidative enzyme on the met-Hb formation and lipid peroxidation should be clarified further. 


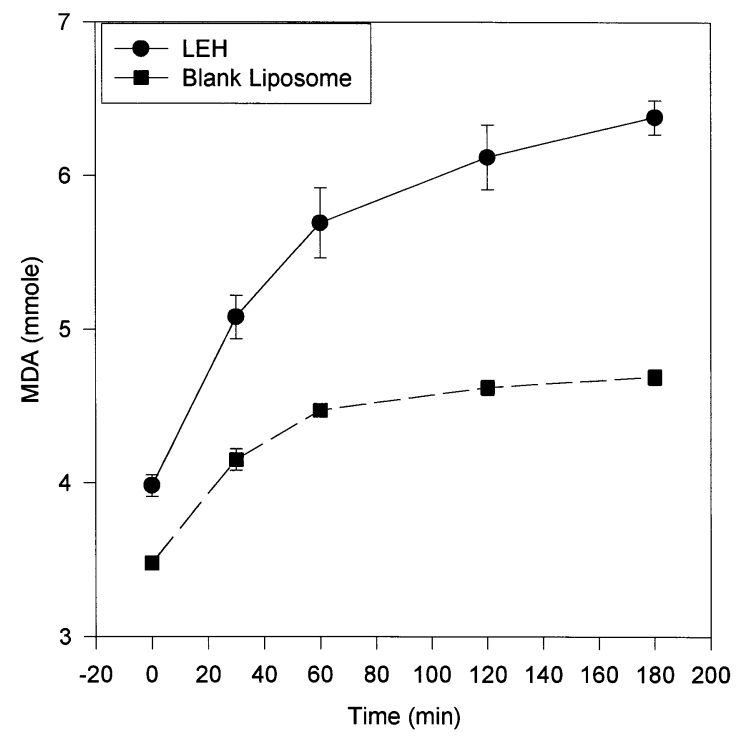

Fig. 5. Difference of peroxidation between LEH and blank liposome under the $1 \mu \mathrm{M} \mathrm{NaClO}_{4}$ with $1.5 \mathrm{mM} \mathrm{Fe}^{2+}$ in the PBS. $(n=4)$ (Data are the average of four consecutive experiments, mean \pm S.D.)

\section{Conclusion}

Natural unsaturated PCs are susceptible to peroxidation. Although $\alpha$-tocopherol is a good antioxidant, it may increase the possibility of liposome aggregation and/or fusion. Replacement of unsaturated PC with saturated PC instead of adding $\alpha$-tocopherol would provide inhibition of peroxidation. Increasing the H-PC content in the liposome formulation will effectively reduce the peroxidation. The stability of liposome was dependent on both $\alpha$-tocopherol and H-PC to PC ratio. Encapsulating hemoglobin in the liposome would promote the lipid peroxidation.

\section{Acknowledgements}

This research was supported by the National Science Council, Taipei, Taiwan, under Contract No. NSC87-2314-B-002-312-M08.

\section{References}

Brandl, M., Gregoriadis, G., 1994. Entrapment of haemoglobin into liposomes by dehydration-rehydration method: vesicle characterization and in vivo behavior. Biochim. Biophys. Acta 1196, 65-75.

Chang, T.M.S. (Ed.), 1993. Blood Substitutes and Oxygen Carriers. Marcel Dekker, New York.

Chung, T.W., Hwang, G.H., Chen, W.K., Lee, C.J., 1997. The effects of plasma and shear force on the stability of liposome encapsulated haemoglobin - in vitro testing. J. Chin. Inst. Chem. Eng. 28, 407-413.

Deshpande, V., Beissinger, R.L., 1993. Liposome-encapsulated hemoglobin using film hydration processing to form artificial red blood cells. Art. Cells, Blood Subs. Immob. Biotech. 21, 135-151.

Farmer, M.C., Gaber, B.P., 1987. Liposome-encapsulated hemoglobin as an artificial oxygen-carrying system. Methods Enzymol. 149, 184-200.

Grit, M., Zuidam, N.J., Underberg, W.J.M., Crommelin, D.J.A., 1993. Hydrolysis of partially saturated egg phosphatidylcholine in aqueous liposome dispersion and the effect of cholesterol incorporation on hydrolysis kinetics. J. Pharm. Pharmacol. 45, 490-495.

Hunt, C.A., Tsang, S., 1981. $\alpha$-tocopherol retards auto-oxidation and prolongs the shelf-life of liposome. Int. J. Pharm. 8, 101-110.

Hunt, C.A., Burnette, R.R., MacGregor, R.D., Strubbe, A.E., Lau, D.T., Taylor, N., Kawada, H.S., 1985. Synthesis and evaluation of a prototypal artificial red cells. Science 230, $1165-1168$.

John, M.C.G., 1986. Iron promoters of the fenton reaction and lipid peroxidation can be released from haemoglobin by peroxides. FEBS 201, 291-295.

Kan, P., Lee, C.J., 1994. Application of aqueous two-phase systems in separation/purification of stroma free hemoglobin from animal blood. Art. Cells Blood Subs. Immob. Biotech. 22, 641-649.

Konings, A.W.T., 1984. Lipid peroxidation in liposomes. In: Gregoriadis, D. (Ed.), Liposome Technology, vol. 1. CRC Press, Boca Raton, FL, pp. 139-161

Lang, J., Vigo-Pelfrey, C., Martin, F., 1990. Liposomes composed of partially hydrogenated egg phosphatidylcholine: fatty acid composition, thermal phase behavior and oxidative stability. Chem. Phys. Lipids 53, 91-101.

Lee, C.J., Kan, P., 1995. Hemoglobin purification. U.S. Patent 5407579.

New, R.R.C., 1990. Liposomes: A Practical Approach. Oxford University Press, New York, pp. 100-101.

Panasenko, O.M., Evgina, S.A., Driomina, E.S., Sharov, V.S., Sergienko, V.I., Vladimirov, Y.A., 1995. Hypochlorite induces lipid peroxidation in blood lipoproteins and phopholipid liposomes. Free Radic. Biol. Med. 19, 133140.

Rollwagen, F.M., Gafney, W.C.M., Pacheco, N.D., Davis, T.A., Hickey, T.M., Nielsen, T.B., Rudolph, A.S., 1996. Multiple response to administration of liposome-encapsu- 
lated hemoglobin (LEH): effect on hematopoiesis and serum IL-6 levels. Exp. Hematol. 24, 429-436.

Smolen, J.E., Shohet, S.B.J., 1974. Permeability changes induced by peroxidation in liposomes prepared from human erythrocyte lipids. J. Lipids Res. 15, 273-280.

Uchiyama, M., Michara, M., 1978. Determination of malonaldehyde precursor in tissues by thiobartituric acid test. Anal. Biochem. 86, 271-278.
Winterbourn, C.C., 1995. Toxicity of iron and hydrogen peroxide: fenton reaction. Toxicol. Lett. 82/83, 969974.

Zheng, S., Zheng, Y., Beissinger, R., Martin, F.J., 1994. Efficacy, physical properties and pharmacokinetics of sterically stabilized liposome-encapsulated hemoglobin. Art. Cells Blood Subs. Immob. Biotech. 22, $487-$ 501 . 